\title{
Can Ki-67 Play a Role in Prediction of Breast Cancer Patients' Response to Neoadjuvant Chemotherapy?
}

\author{
Juhasz-Böss Ingolf, ${ }^{1}$ Mavrova Russalina, ${ }^{1}$ Moga Simona, ${ }^{1}$ Radosa Julia, ${ }^{1}$ Schmidt Gilda, \\ Rainer M. Bohle, ${ }^{2}$ Hasenfus Andrea, ${ }^{2}$ Solomayer Erich, ${ }^{1}$ and Herr Daniel ${ }^{1}$ \\ ${ }^{1}$ Department of Obstetrics and Gynecology, Homburg University Medical Centre, 66421 Homburg, Germany \\ ${ }^{2}$ Department of Pathology, Homburg University Medical Centre, 66421 Homburg, Germany
}

Correspondence should be addressed to Juhasz-Böss Ingolf; ingolf.juhasz-boess@uks.eu

Received 13 January 2014; Accepted 11 February 2014; Published 25 March 2014

Academic Editor: Peter A. Fasching

Copyright (C) 2014 Juhasz-Böss Ingolf et al. This is an open access article distributed under the Creative Commons Attribution License, which permits unrestricted use, distribution, and reproduction in any medium, provided the original work is properly cited.

\begin{abstract}
Background. Currently the choice of breast cancer therapy is based on prognostic factors. The proliferation marker Ki-67 is used increasingly to determine the method of therapy. The current study analyses the predictive value of Ki-67 in foreseeing breast cancer patients' responses to neoadjuvant chemotherapy. Methods. This study includes patients with invasive breast cancer treated between 2008 and 2013. The clinical response was assessed by correlating Ki-67 to histological examination, mammography, and ultrasonography findings. Results. The average Ki-67 value in our patients collectively $(n=77)$ is $34.9 \pm 24.6 \%$. The average Ki-67 value is the highest with $37.4 \pm 24.0 \%$ in patients with a pCR. The Ki-67 values do not differ significantly among the 3 groups: pCR versus partial pathological response versus stable disease/progress $(P=0.896)$. However, Ki-67 values of patients with luminal, Her2 enriched, and basal-like cancers differed significantly from each other. Furthermore, within the group of luminal tumors Ki67 values of patients with versus without pCR also differed significantly. Conclusion. Our data shows that the Ki-67 value predicts the response to neoadjuvant chemotherapy as a function of the molecular subtype, reflecting the daily routine concerning Ki-67 and its impressing potential and limitation as a predictive marker for neoadjuvant chemotherapy response.
\end{abstract}

\section{Introduction}

Breast cancer is the most diagnosed cancer in women. However, breast cancer mortality rate in industrialised western countries has decreased in the last decades [1, 2]. Early diagnosis and effective therapies contribute greatly to this decrease in mortality rate [3]. Currently the choice of therapy is based on prognostic factors. Different already known prognostic factors such as histological tumour type, tumour size, nodal status, grade, age, and estrogen receptor (ER) status and the proliferation marker Ki-67 influence the type of therapy decision [4]. The clinical use of these factors aims at identifying patients with an unfavourable prognosis and at improving the treatment according to the individual risk (recurrence and mortality). The use of this paradigm over the past three years has led to notable therapy improvement $[5,6]$.

Moreover, chemotherapy-indication is based on prognostic factors. All patients with an indication for adjuvant chemotherapy can be offered a neoadjuvant treatment [710]. A neoadjuvant chemotherapy regiment offers a lot of advantages compared to adjuvant treatment. The response to chemotherapy and therefore also its effectiveness can be better monitored, thus potentially increasing patient compliance. In addition, the use of neoadjuvant cytotoxic treatment may increase the rate of breast conserving therapy and reduce the extent of surgery [8].

Another potential prognostic marker is pathologic complete response (pCR). In many neoadjuvant studies, patients who achieve a pCR showed a better long-term outcome [11, 12]. A pooled analysis of seven randomized trials, including 6,377 patients, showed a significant difference in disease-free survival (DFS) between patients with pCR (ypT0/N0) and patients without pCR. The overall survival (OS) was also better for the former patients. Furthermore, this study shows that pCR is only in highly proliferating breast cancers, like triple negative breast cancer (TNBC), HER2 enriched (HER2 
positive plus ER negative), or luminal B/HER2-negative tumours, a good prognostic value, whereas in luminal $A$ and luminal B (ER plus HER2 positive) tumours the pCR is not able to discriminate between good and poor prognosis [13, 14]. In addition to the pCR after neoadjuvant chemotherapy, the proliferation marker Ki-67 is not only a prognostic but also a predictive value.

Ki-67 is a nuclear antigen identified in 1983 which is present in the nuclei of cells in all phases of the cell cycle as well as in mitosis, but quiescent cells in the $G_{0}$ phase do not express it [15-17]. In fact it is the most common marker used in clinical practice. Kwok et al. showed in 2010 that the proliferation marker $\mathrm{Ki}-67$ in needle core biopsy showed better concordance with haematoxylin and eosin mitotic count in surgical excision specimen than routine haematoxylin and eosin mitotic count in needle core biopsy [18]. Relating to neoadjuvant chemotherapy and its response, it has been found that a high level of proliferation activity has predictive value [19]. Fasching et al. showed in 2011 that the response of neoadjuvant chemotherapy in patients with a high Ki-67 level (>30\%) was better than in other tumours [20]. Moreover, after a neoadjuvant chemotherapy Ki-67 is still able to function as prognostic marker. Patients with high Ki-67 values in the residual tumour after chemotherapy had a poorer outcome regarding recurrence and mortality. These high risk patients may require further systemic therapy. However, despite these positive qualities $\mathrm{Ki}-67$ is a regular topic of discussion due to its cut-off values and the intra- and interlaboratory reproducibility.

Therefore, the current study was performed in order to analyze retrospectively the predictive value of Ki-67 in prediction of responses of breast cancer patients to neoadjuvant chemotherapy treatment conducted in a German university hospital.

\section{Patients, Material, and Methods}

This retrospective single-center study is composed solely of patients treated by neoadjuvant chemotherapy for invasive breast cancer at a tertiary university center (Saarland University Hospital) between January 2008 and December 2013. The inclusion criteria are that the performance of initial core needle biopsy leading to histopathological diagnosis and surgery following neoadjuvant chemotherapy must be performed at the Department of Gynaecology and Obstetrics of Saarland University Hospital. The exclusion criteria are incomplete data, histopathologic diagnosis and surgery performed at a different institution, and patients with metastasis at the time of initial diagnosis.

Clinical data were obtained using medical records and original pathology reports and collected in an Excel database (Microsoft Corporation, Redmond, WA, USA). The following parameters were assessed: patient's age, tumor size (defined as sonographic diameter ( $\mathrm{mm}$ ) on diagnosis), initial tumor stage and nodal status according to TMN classification, histologic subtype, estrogen receptor status, progesterone status, HER2 status, grading and proliferation status as assessed by Ki-67 staining, neoadjuvant chemotherapy regime and neoadjuvant targeted therapy, posttherapeutic sonographic tumor diameter $(\mathrm{mm})$, posttherapeutic histologic tumor diameter $(\mathrm{mm})$, and posttherapeutic tumor stage and nodal status. Histopathological regression was classified using the semiquantitative scoring system according to Sinn from 0 to 4 $(0=$ no effect, $1=$ resorption and tumor sclerosis, $2=$ minimal residual invasive tumor $[<0.5 \mathrm{~cm}], 3=$ residual noninvasive tumor only, and $4=$ no tumor detectable). A regression grade of four according to Sinn was defined as pathologic complete response (pCR) and a regression grade from two upwards was defined as pathologic partial remission (pPR).

Clinical response was assessed based on a physical examination, mammography, and ultrasonography according to the Response Evaluation Criteria in Solid Tumors (RECIST) [12]. A clinical complete response (cCR) was defined as the disappearance of all known lesions; a clinically partial response ( $\mathrm{CPR}$ ) was defined as $\mathrm{a} \geq 30 \%$ reduction in the sum of the longest diameter (LD) of the primary lesion; progressive disease (PD) was defined as a $\geq 20 \%$ increase in the sum of the LD of the primary lesion; and stable disease (SD) was defined as neither sufficient shrinkage to qualify for $\mathrm{CPR}$ nor sufficient increase to qualify for PD. The study protocol was approved by the hospital's ethics board and informed consents were obtained from patients in the study.

All histopathological parameters included were derived from the original pathology reports. Tumor tissue was neutral-buffered, formalin-fixed, and paraffin-embedded. Staining of the pretreatment core biopsies was performed using monoclonal rabbit antibodies against estrogen receptor-alpha (clone SP1, DCS Hamburg, Germany), monoclonal rabbit antibody against the progesterone receptor (clone SP2, DCS Hamburg, Germany), and monoclonal antibody against Ki-67 (clone MIB-1, DAKO, Glostrup, Denmark), each according to the manufacturer's instructions using a slide stainer (BenchMark ULTRA, Ventana Medical Systems, Arizona, USA). For evaluation of $\mathrm{Ki}-67$, areas with the highest $\mathrm{Ki}-67$ labeling were investigated. Visualization of antigenic sites was performed using the DakoEnVision kit (Hamburg, Germany). For staining of Her2/neu rabbit antibody was used (A0485, DAKO, Glostrup, Denmark). Her2 status was given on a scale from 0 to $3+$. A score of 0 or $1+$ was regarded as Her2 negative and a score of $3+$ as positive. In case of intermediate score $(2+)$ samples were tested for gene amplification using a Her2 fluorescence in situ hybridization kit (Zytolight, SPEC HER2/CEN17 Dual Color Probe, Zyto Vision Ltd., Bremerhaven, Germany). Hereby gene copy numbers of HER2 and centromeres of the corresponding chromosome 17 were retrieved. A HER2/CEN17 ratio of $>2.2$ was considered as amplification of HER2. Cases with a ratio between 1.8 and 2.2 were reevaluated by repeating the staining procedure [12]. Scoring was performed according to standardized protocols by specialized pathologist at the Department of Pathology, Saarland University Hospital.

We only analysed 77 patients in this study, since for the missing cases Ki-67 data was not available. Data was collected in an EXCEL database (Microsoft Corporation, Redmond, WA, USA) and statistical calculations were performed with SPSS (SPSS Inc. Chicago, IL, USA). One way analysis of 
TABLE 1: Patient characteristics. Discrete data are given as numbers, continuous as the mean \pm standard deviation.

\begin{tabular}{|c|c|}
\hline Parameter & Value \\
\hline Total number of patients & 77 \\
\hline Age at first diagnosis (years) & $57.8 \pm 10.9$ \\
\hline Tumor diameter (initial) & $31.1 \pm 13.6$ \\
\hline \multicolumn{2}{|l|}{ Histotype } \\
\hline Invasive ductal & $70(90.9 \%)$ \\
\hline Invasive lobular & $6(7.7 \%)$ \\
\hline Others & $1(1.3 \%)$ \\
\hline \multicolumn{2}{|l|}{ Tumor stage (initial) } \\
\hline la & - \\
\hline $1 b$ & $1(1.3 \%)$ \\
\hline $1 \mathrm{c}$ & $6(7.7 \%)$ \\
\hline 2 & $49(63.6 \%)$ \\
\hline 3 & $5(6.5 \%)$ \\
\hline 4 & $16(20.7 \%)$ \\
\hline \multicolumn{2}{|l|}{ Nodal status } \\
\hline Negative & $25(32.4 \%)$ \\
\hline Positive & $52(67.5 \%)$ \\
\hline \multicolumn{2}{|l|}{ Metastasis } \\
\hline Negative & $77(100 \%)$ \\
\hline Positive & - \\
\hline \multicolumn{2}{|l|}{ Tumor grade } \\
\hline 1 & $1(1.3 \%)$ \\
\hline 2 & $37(48 \%)$ \\
\hline 3 & $39(50.6 \%)$ \\
\hline $\mathrm{Ki}-67$ & $34.9 \pm 24.6$ \\
\hline \multicolumn{2}{|l|}{ Estrogen receptor (ER) } \\
\hline Negative & $35(45.4 \%)$ \\
\hline Positive & $42(54.5 \%)$ \\
\hline \multicolumn{2}{|l|}{ Progesterone receptor (PR) } \\
\hline Negative & $40(51.9 \%)$ \\
\hline Positive & $37(48 \%)$ \\
\hline \multicolumn{2}{|l|}{ Her2 receptor } \\
\hline Negative & $57(74 \%)$ \\
\hline Positive & $20(25.9 \%)$ \\
\hline “Triple negative" & $23(29.8 \%)$ \\
\hline
\end{tabular}

variance (ANOVA) and paired samples $t$-test were used for analysis. A $P$ value $<0.05$ was considered to indicate statistical significance. Data is reported as mean \pm standard error.

\section{Results}

More than 1,000 patients with breast cancer were treated between 2008 and 2013 at the University Hospital of the Saarland. A total of 114 patients received neoadjuvant chemotherapy during this period. The complete medical records, including patient characteristics, tumor characteristics, treatment data, and epidemiological data, and furthermore a Ki-67 determination from 77 patients were analyzed retrospectively. The results of the analysis of these 77 patients are presented in this section.
TABle 2: Therapeutic and postoperative characteristics. Discrete data are given as numbers, continuous as the mean \pm standard deviation.

\begin{tabular}{lc}
\hline Parameter & Value \\
\hline Total number of patients & 77 \\
Neoadjuvant chemotherapy & \\
EC/DOC & $61(79.2 \%)$ \\
TAC & $11(14.2 \%)$ \\
Others & $5(6.4 \%)$ \\
Endocrine therapy & - \\
Neoadjuvant targeted therapy & \\
Trastuzumab & $15(19.4 \%)$ \\
Lapatinib & $3(3.8 \%)$ \\
Trastuzumab + pertuzumab & $2(2.5 \%)$ \\
Bevacizumab & $6(7.7 \%)$ \\
None & $51(66.2 \%)$ \\
Posttherapeutic sonographic tumor diameter $(\mathrm{mm})$ & $12.6 \pm 10.0$ \\
Postoperative histologic tumor diameter (mm) & $15.7 \pm 17.1$ \\
Postoperative tumor stage & \\
0 & $20(25.9 \%)$ \\
la & $9(11.6 \%)$ \\
$1 b$ & $7(9 \%)$ \\
$1 \mathrm{c}$ & $18(23.3 \%)$ \\
2 & $15(19.4 \%)$ \\
3 & $6(7.7 \%)$ \\
Grade of regression (Sinn) & $2(2.5 \%)$ \\
0 & \\
1 & $28(36.3 \%)$ \\
3 & $22(28.5 \%)$ \\
Unknown & $2.5 \%)$ \\
\hline & $4(5.1 \%)$ \\
\hline
\end{tabular}

The average patients' age was 57,8 years old when receiving the initial diagnosis of breast cancer. Tumor characteristics including tumor entity, the initial size of the tumor, the TNM status, the Ki-67 determination, the hormone receptor, and Her2 status were recorded, as shown in Table 1. All patients received neoadjuvant chemotherapy; none received a primary hormone therapy. Information about the administered chemotherapy and in some cases an additional targeted therapy depending on the receptor status is presented in Table 2. Table 2 also includes information about the average tumor size in ultrasound imaging after the neoadjuvant chemotherapy, the average size according to pathological assessment, the TNM status after the treatment, and the pathological response as in Sinn's assessment. The development of tumor size, before the start and after the completion of the neoadjuvant treatment is shown in Figure 1. The average Ki-67 value in our patients collectively was $34,9 \pm$ $24,6 \%$ (range 1-90\%). A correlation between the Ki-67 value and a response to the neoadjuvant chemotherapy is illustrated in Figure 2. Twenty patients showed a complete pathological response (pCR), thirty-eight patients showed a partial either 


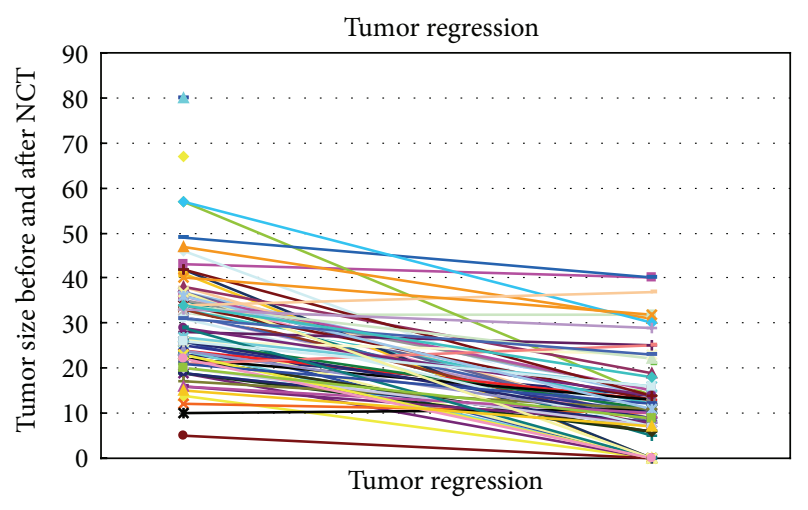

FIGURE 1: Tumor response, as measured from the maximum initial sonographic diameter and the maximum histological diameter after neoadjuvant chemotherapy and surgery. Data is presented for the values of $n=77$ patients.

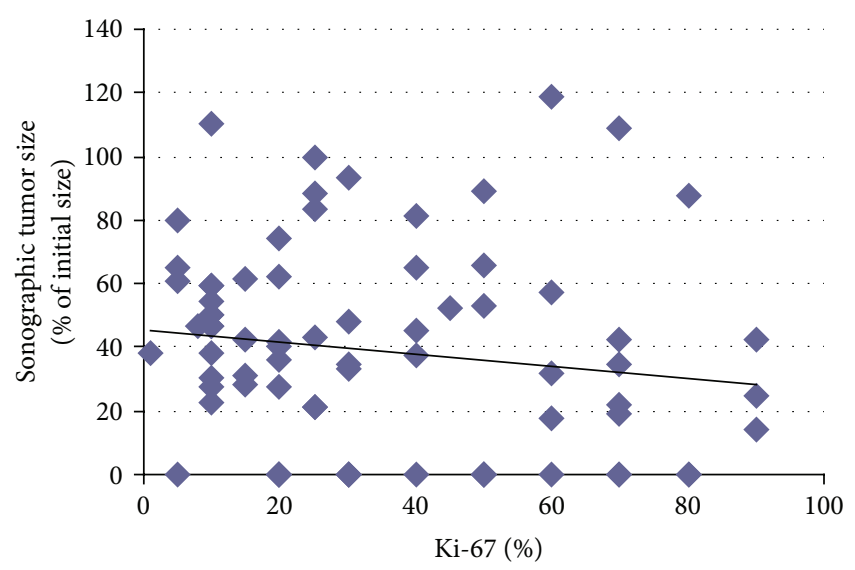

FIGURE 2: Ki-67 values and sonographic response after neoadjuvant chemotherapy. Tumor sizes are presented in \% of the initial sizes.

clinical or pathological response, and seventeen patients had a stable disease or a progress of the disease after finishing the neoadjuvant chemotherapy (Figure 3). In the patient collective with a complete pathological response, the average Ki-67 value was the highest with $37.4 \pm 24.0 \%$. Patients with a partial pathological response showed an average Ki-67 value of $34.7 \pm 25.5 \%$. Patients with stable disease or rather progress had an average Ki-67 value of $33.8 \pm 25.8 \%$. The Ki-67 values do not differ significantly among the 3 groups $(P=0.896)$ as illustrated in Figure 3.

Additionally we divided our patients collectively into 3 groups depending on the cut-off values for Ki-67. We tried to find any differences in initial tumor size and tumor characteristics and investigated the predictive value of $\mathrm{Ki}-67$ for the success of neoadjuvant chemotherapy by correlating it to the pathological response (Table 3). Group A $(n=20)$ represented a group of tumors showing low Ki-67 values $(\leq 15 \%)$, group B $(n=37)$ included tumors with an average Ki67 value between 15 and $50 \%$, and group C $(n=18)$ consisted of tumors with high average Ki-67 values of more than $50 \%$. There were no significant differences between the 3 groups

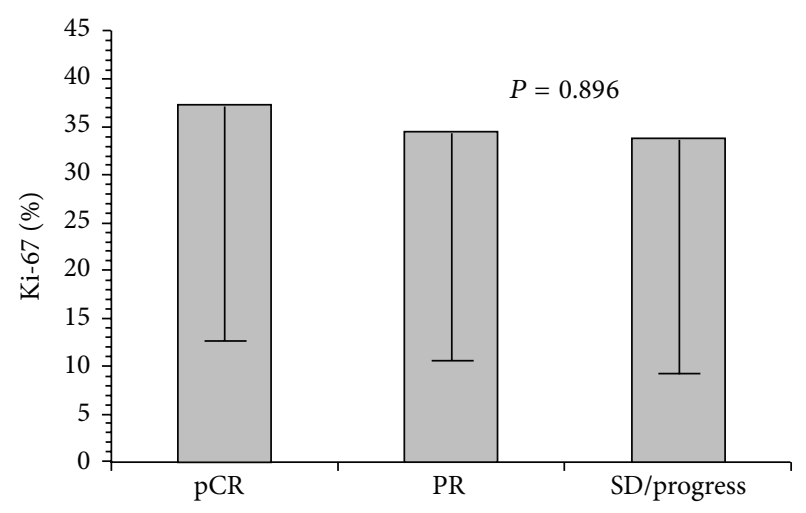

FIGURE 3: Ki-67 in dependence of the pathological response. pCR = complete pathological response, $\mathrm{PR}=$ partial either clinical or pathological response, and $\mathrm{SD} /$ progress = stable disease or a progress (mean \pm standard deviation).

concerning initial tumor diameter, postoperative histologic tumor diameter, change from initial to posttreatment tumor size in ultrasound imaging, pathological assessment, grade of regression according to Sinn, and the number of patients with a complete pathological response ( $\mathrm{pCR}$ ).

However, subdividing the patients according to the molecular subtype of their cancer (Luminal, Her2 enriched, and basal-like), we detected significant differences of Ki67 between those groups: Ki-67 values of triple negative cancers were $60.4 \pm 18.3 \%$, Her-2 positive cancers were 25.4 $\pm 12.6 \%$, and luminal tumors $22 \pm 19.5 \%(P<0.0001)$. In addition, within the group of luminal tumors Ki-67 values of patients with versus without pCR differed significantly: patients receiving a pCR presented with $\mathrm{Ki}-67$ values of 50 $\pm 36.5 \%$ versus $18.1 \pm 12.9 \%(P=0.001)($ Table 4$)$.

\section{Discussion}

In this retrospective study, 77 breast cancer patients receiving neoadjuvant chemotherapy were analysed concerning Ki67 and its impact as predictive marker for chemotherapy response. We found a trend towards the highest Ki-67 values in patients achieving a pCR as compared to patients with partial response, stable disease, or progress. However, the observed differences are not significant. In addition, three different groups referring to low, medium, or high levels of Ki-67 have been formed and analysed by correlating the groups to the pathological response. Again no significant differences could be found.

The average Ki-67 value in our population was 34,9\% ranging from 1 to $90 \%$. Even in the pCR group with an average $\mathrm{Ki}-67$ of $37,4 \%$ the values ranged $\pm 24 \%$. This average value showed a higher trend as compared to the group with partial response, stable disease, or progress. Although the St. Gallen Consensus 2013 recommended the use of Ki-67 as additional factor in order to distinguish the large group of receptor positive breast cancers in luminal $\mathrm{A}$ and $\mathrm{B}$, there are several problems concerning the detection of Ki-67. One of the problems of $\mathrm{Ki}-67$ use is the large inter- and intraobserver 
TABLE 3: Therapeutical aspects as a function of Ki-67.

\begin{tabular}{|c|c|c|c|c|}
\hline Parameter & Ki- $67 \leq 15 \%$ & Ki-67 16-50\% & Ki-67>50\% & $P$ \\
\hline Number of patients & 22 & 37 & 18 & \\
\hline Initial sonographic tumor diameter (mm) & $29.3 \pm 14.0$ & $32.2 \pm 15.0$ & $31.1 \pm 10.4$ & 0.738 \\
\hline Postoperative histologic tumor diameter ( $\mathrm{mm}$ ) & $14.6 \pm 9.9$ & $18.6 \pm 21.7$ & $11.0 \pm 15.3$ & 0.312 \\
\hline Change ( $\%$ from initial tumor size) & $57.9 \pm 44.9$ & $52.0 \pm 66.2$ & $39.4 \pm 50.7$ & 0.590 \\
\hline Sonographic change (\% from initial tumor size) & $47.6 \pm 22.2$ & $38.2 \pm 32.8$ & $34.4 \pm 37.0$ & 0.373 \\
\hline Grade of regression (Sinn) & $1.7 \pm 1.1$ & $2.2 \pm 1.2$ & $2.1 \pm 1.4$ & 0.310 \\
\hline Number of patients with pCR & $4(18.1 \%)$ & $10(27 \%)$ & $6(33.3 \%)$ & \\
\hline
\end{tabular}

TABLE 4: Ki-67 as a function of molecular subtypes.

\begin{tabular}{lcccc}
\hline & Total & pCR yes & pCR no & \\
\hline Triple negative & $n=23$ & $n=5$ & $n=19$ & $61 \pm 18.7$ \\
& $60.4 \pm 18.3$ & $57 \pm 18.5$ & $n=9$ & $P=0.651$ \\
Her2 positive & $n=20$ & $n=11$ & $27.2 \pm 15.6$ & \\
& $25.4 \pm 12.6$ & $23.9 \pm 10.2$ & $n=29$ & $P=0.576$ \\
Luminal & $n=33$ & $n=4$ & $18.1 \pm 12.9$ & $P=\mathbf{0 . 0 0 1}$ \\
\hline
\end{tabular}

variation. In 2013 Polley et al. compared the Ki-67 levels in eight of the world's most experienced laboratories and observed a large variation among those laboratories [21]. The most commonly used assay to assess Ki-67 is immunohistochemical (IHC) staining with the MIB-1 antibody. Different groups used different antibodies on paraffin sections after antigen retrieval, such as MM-1, Ki-S5, SP-6, and MIB-1. This might be one reason for the considerable interlaboratory variability. Also, intraobserver variability is a highly discussed issue. The Ki-67 score is defined as the percentage of total number of tumor cells with nuclear staining. Some pathologists estimate the percentage of nuclei staining; others count several hundred nuclei in different areas of tumors to give an overall average index. Therefore more and more automated readers are used. Computer-assisted image analysis can raise the reproducibility of Ki-67 assessment [22], but it has a limited capacity of excluding normal stromal/inflammatory cells [23]. Also, tissue microarray technology has been introduced; its reliability and reproducibility were proven in studies [24]. A standardization of Ki-67 pathological assessment has not yet been accomplished [25]. This lack of consistency across laboratories has thus far limited Ki-67's value. The International Ki-67 in Breast Cancer Working Group was assembled to devise a strategy to harmonize Ki-67 analysis and increase scoring concordance [26].

The above discussed limitations concerning the determination of Ki-67 must be taken into account when discussing our results. However, although the detection and quantification of Ki-67 is difficult our observation of a higher average Ki-67 in the group of patients achieving pCR is in line with several findings of other groups. This concerns also a large number of studies with a neoadjuvant setting. The usefulness of $\mathrm{Ki}-67$ in predicting response and outcome is explored by assessing pretreatment and posttreatment levels of tumor Ki-67 expression in neoadjuvant chemotherapy.
Unfortunately, only few of those studies are randomized [27]. Most neoadjuvant chemotherapy studies only perform a univariate analysis looking at response as an outcome [28]. These four studies found $\mathrm{Ki}-67$ to be a predictive marker for either clinical and/or pathological response but only few authors were able to demonstrate $\mathrm{Ki}-67$ as an independent predictor for $\mathrm{pCR}$ and overall survival in multivariate models [20].

It has to be declared that a few studies report no correlation between $\mathrm{Ki}-67$ and response to neoadjuvant chemotherapy [29], as we were also not able to find at least a significant correlation between Ki-67 and the response after neoadjuvant chemotherapy. However, since a trend towards response could be observed, it has to be assumed that the lack of significance is due to the relatively small number of patients and the retrospective character of our analysis. In addition, the explanatory power of our data is constricted since we do not provide a multicentric assessment.

In the next step, we subdivided our population into three groups depending on the cut-off levels for Ki-67 $(\leq 15 \%$, $15-50 \%$, and $>50 \%$ ). Again the response after neoadjuvant chemotherapy has been analysed between those groups. However, we were not able to detect any significant differences. This issue addresses another major problem: the varying definition of cut-off values for Ki-67 across the different studies. Klintman et al., for example, uses a Ki-67 cut-off of $\leq 20 \%$. The St. Gallen Consensus in 2009 classified tumors as low, intermediate, and highly proliferating according to the value of Ki-67 labeling index of $\leq 15 \%, 16-30 \%$, and $>30 \%$ [30]. Fasching et al. and Cheang et al. used a cut-off point for Ki-67 for more than $13 \%$ positively stained cells [20]. Denkert et al. in 2013 even pretends that Ki-67 is a significant predictive and prognostic marker over a wide range of cut points, suggesting that data-derived cut point optimisation might not be possible [31]. Nevertheless, Ki-67 may be an 
important marker regarding the molecular cancer subtypes. We found that Ki-67 values of patients with Luminal, Her2 enriched and basal-like cancers differed significantly from each other. Furthermore, within the group of luminal tumors Ki-67 values of patients with versus without pCR differed significantly. This data is in line with Fasching et al. [20], who found that patients with luminal cancers and pCR have significantly higher Ki-67 values as compared to those without pCR. In our population, patients with triple negative cancer also had the highest levels of Ki-67. However, in contrast to the data of Fasching et al. we did not observe any differences between the group with and without pCR. This might be due to the relatively small subgroup consisting of only 23 patients.

In conclusion, our data shows that the Ki-67 value predicts the response to neoadjuvant chemotherapy in breast cancer patients as a function of the molecular subtype reflecting the daily routine concerning Ki-67 and its impressing chances and yet also its limitations as predictive marker for neoadjuvant chemotherapy response.

\section{Conflict of Interests}

The authors declare that there is no conflict of interests regarding the publication of this paper.

\section{References}

[1] R. Peto, J. Boreham, M. Clarke, C. Davies, and C. Berai, "UK and USA breast cancer deaths down $25 \%$ in year 2000 at ages 20-69 years," The Lancet, vol. 355, no. 9217, p. 1822, 2000.

[2] N. Eisemann, A. Waldmann, and A. Katalinic, "Epidemiology of breast cancer-current figures and trends," Geburtsh Frauenheilk, vol. 73, no. 2, pp. 130-135, 2013.

[3] D. A. Berry, K. A. Cronin, S. K. Plevritis et al., "Effect of screening and adjuvant therapy on mortality from breast cancer," The New England Journal of Medicine, vol. 353, no. 17, pp. 1784-1792, 2005.

[4] M. Schmidt, P. A. Fasching, M. W. Beckmann, and H. Kolbl, "Biomarkers in breast cancer-an update," Geburtsh Frauenheilk, vol. 72, no. 9, pp. 819-832, 2012.

[5] C. Melcher, C. Scholz, B. Jäger, C. Hagenbeck, B. Rack, and W. Janni, "Breast cancer: state of the art and new findings," Geburtshilfe und Frauenheilkunde, vol. 72, no. 3, pp. 215-224, 2012.

[6] D. Luftner, M. P. Lux, N. Maass et al., "Advances in breast cancer-looking back over the year," Geburtsh Frauenheilk, vol. 72, no. 12, pp. 1117-1129, 2012.

[7] H. D. Bear, S. Anderson, R. E. Smith et al., "Sequential preoperative or postoperative docetaxel added to preoperative doxorubicin plus cyclophosphamide for operable breast cancer: national surgical adjuvant breast and bowel project protocol B27," Journal of Clinical Oncology, vol. 24, no. 13, pp. 2019-2027, 2006.

[8] M. Kaufmann, G. Von minckwitz, H. D. Bear et al., "Recommendations from an international expert panel on the use of neoadjuvant (primary) systemic treatment of operable breast cancer: new perspectives 2006," Annals of Oncology, vol. 18, no. 12, pp. 1927-1934, 2007.
[9] G. Von Minckwitz, G. Raab, A. Caputo et al., "Doxorubicin with cyclophosphamide followed by docetaxel every 21 days compared with doxorubicin and docetaxel every 14 days as preoperative treatment in operable breast cancer: the GEPARDUO study of the German Breast Group," Journal of Clinical Oncology, vol. 23, no. 12, pp. 2676-2685, 2005.

[10] A. Wöckel and R. Kreienberg, "First revision of the German S3 guideline "diagnosis, therapy, and follow-up of breast cancer"', Breast Care, vol. 3, no. 2, pp. 82-86, 2008.

[11] J. S. Mieog, J. A. van der Hage, and C. J. van de Velde, "Preoperative chemotherapy for women with operable breast cancer," Cochrane Database of Systematic Reviews, vol. 18, no. 2, 2007.

[12] P. Rastogi, S. J. Anderson, H. D. Bear et al., "Preoperative chemotherapy: updates of national surgical adjuvant breast and bowel project protocols B-18 and B-27," Journal of Clinical Oncology, vol. 26, no. 5, pp. 778-785, 2008.

[13] S. P. Gampenrieder, G. Rinnerthaler, and R. Greil, "Neoadjuvant chemotherapy and targeted therapy in breast cancer: past, present, and future," Journal of Oncology, vol. 2013, Article ID 732047, 12 pages, 2013.

[14] G. Von Minckwitz, M. Untch, E. Nüesch et al., "Impact of treatment characteristics on response of different breast cancer phenotypes: pooled analysis of the German neo-adjuvant chemotherapy trials," Breast Cancer Research and Treatment, vol. 125, no. 1, pp. 145-156, 2011.

[15] D. C. Brown and K. C. Gatter, "Ki67 protein: the immaculate deception?" Histopathology, vol. 40, no. 1, pp. 2-11, 2002.

[16] J. Gerdes, U. Schwab, H. Lemke, and H. Stein, "Production of a mouse monoclonal antibody reactive with a human nuclear antigen associated with cell proliferation," International Journal of Cancer, vol. 31, no. 1, pp. 13-20, 1983.

[17] T. Scholzen and J. Gerdes, “The Ki-67 protein: from the known and the unknown," Journal of Cellular Physiology, vol. 182, pp. 311-322, 2000.

[18] T. C. Kwok, E. A. Rakha, A. H. S. Lee et al., "Histological grading of breast cancer on needle core biopsy: the role of immunohistochemical assessment of proliferation," Histopathology, vol. 57, no. 2, pp. 212-219, 2010.

[19] G. MacGrogan, L. Mauriac, M. Durand et al., "Primary chemotherapy in breast invasive carcinoma: predictive value of the immunohistochemical detection of hormonal receptors, p53, c-erbB-2, MiB1, pS2 and GST $\pi$," British Journal of Cancer, vol. 74, no. 9, pp. 1458-1465, 1996.

[20] P. A. Fasching, K. Heusinger, L. Haeberle et al., "Ki67, chemotherapy response, and prognosis in breast cancer patients receiving neoadjuvant treatment," BMC Cancer, vol. 11, article 486, 2011.

[21] M. Y. Polley, S. C. Leung, and L. M. McShane, "An international ki67 reproducibility study," Journal of the National Cancer Institute, vol. 105, no. 24, pp. 1897-1906, 2013.

[22] A. Vörös, E. Csörgö, and B. Kövári, “The use of digitalimages improves reproducibility of the Ki-67 labeling index as a proliferation marker in breast cancer," Pathology \& Oncology Research. In press.

[23] S. Fasanella, E. Leonardi, C. Cantaloni et al., "Proliferative activity in human breast cancer: Ki-67 automated evaluation and the influence of different Ki-67 equivalent antibodies," Diagnostic Pathology, vol. 6, no. 1, article S7, 2011.

[24] P. Ruiz and H. Witt, "Microarray analysis to evaluate different animal models for human heart failure," Journal of Molecular and Cellular Cardiology, vol. 40, no. 1, pp. 13-15, 2006. 
[25] M. Colozza, A. Sidoni, and M. Piccart-Gebhart, "Value of Ki67 in breast cancer: the debate is still open," The Lancet Oncology, vol. 11, no. 5, pp. 414-415, 2010.

[26] M. Dowsett, T. O. Nielsen, R. A'Hern et al., "Assessment of Ki67 in Breast Cancer: recommendations from the international Ki67 in breast cancer working Group," Journal of the National Cancer Institute, vol. 103, no. 22, pp. 1656-1664, 2011.

[27] G. Von Minckwitz, H.-P. Sinn, G. Raab et al., "Clinical response after two cycles compared to HER2, Ki-67, p53, and bcl-2 in independently predicting a pathological complete response after preoperative chemotherapy in patients with operable carcinoma of the breast," Breast Cancer Research, vol. 10, no. 2, article R30, 2008.

[28] C. Bozzetti, A. Musolino, R. Camisa et al., "Evaluation of HER2/neu amplification and other biological markers as predictors of response to neoadjuvant anthracycline-based chemotherapy in primary breast cancer: the role of anthracycline dose intensity," American Journal of Clinical Oncology: Cancer Clinical Trials, vol. 29, no. 2, pp. 171-177, 2006.

[29] A. Bottini, A. Berruti, A. Bersiga et al., "Relationship between tumour shrinkage and reduction in Ki67 expression after primary chemotherapy in human breast cancer," British Journal of Cancer, vol. 85, no. 8, pp. 1106-1112, 2001.

[30] A. Goldhirsch, W. C. Wood, A. S. Coates, R. D. Gelber, B. Thürlimann, and H.-J. Senn, "Strategies for subtypes-dealing with the diversity of breast cancer: highlights of the St Gallen international expert consensus on the primary therapy of early breast cancer 2011," Annals of Oncology, vol. 22, no. 8, pp. 1736$1747,2011$.

[31] C. Denkert, S. Loibl, and B. M. Müller, "Ki67 levels as predictive and prognostic parameters in pretherapeutic breast cancer core biopsies: a translational investigation in the neoadjuvant GeparTrio trial," Annals of Oncology, vol. 24, no. 11, pp. 27862793, 2013. 


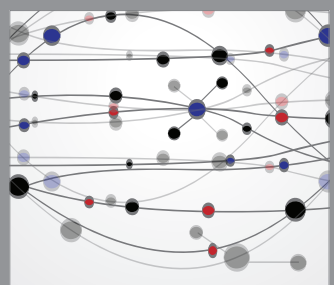

The Scientific World Journal
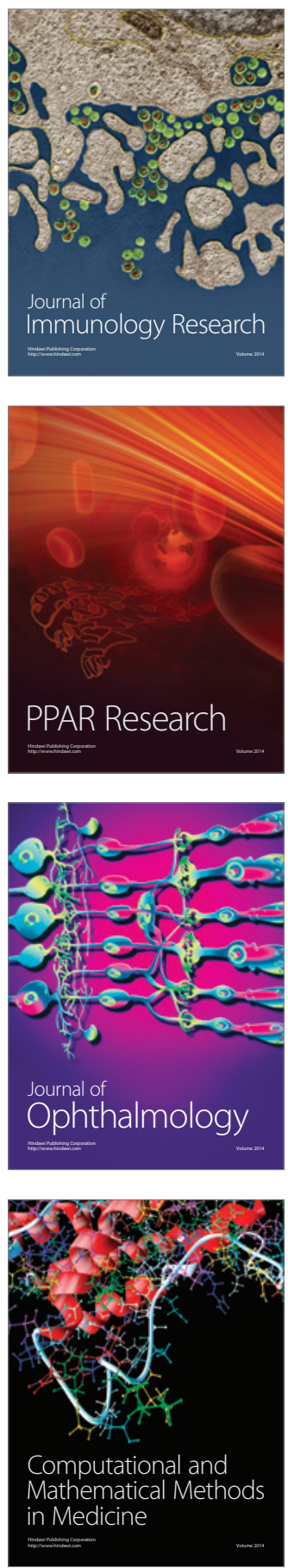

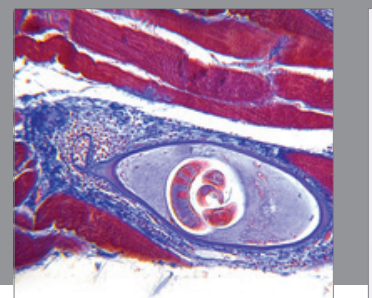

Gastroenterology

Research and Practice
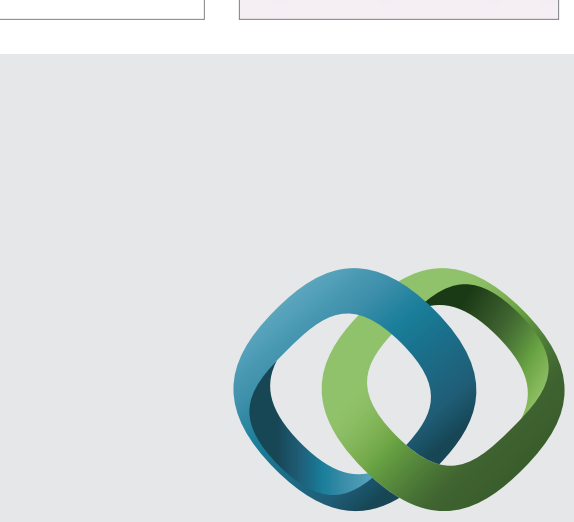

\section{Hindawi}

Submit your manuscripts at

http://www.hindawi.com
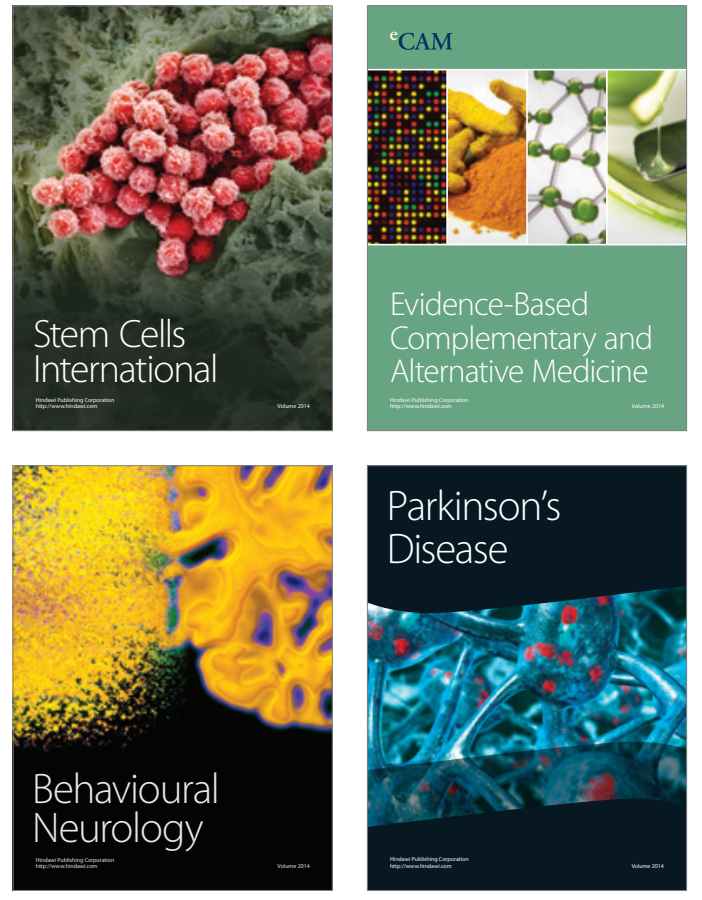
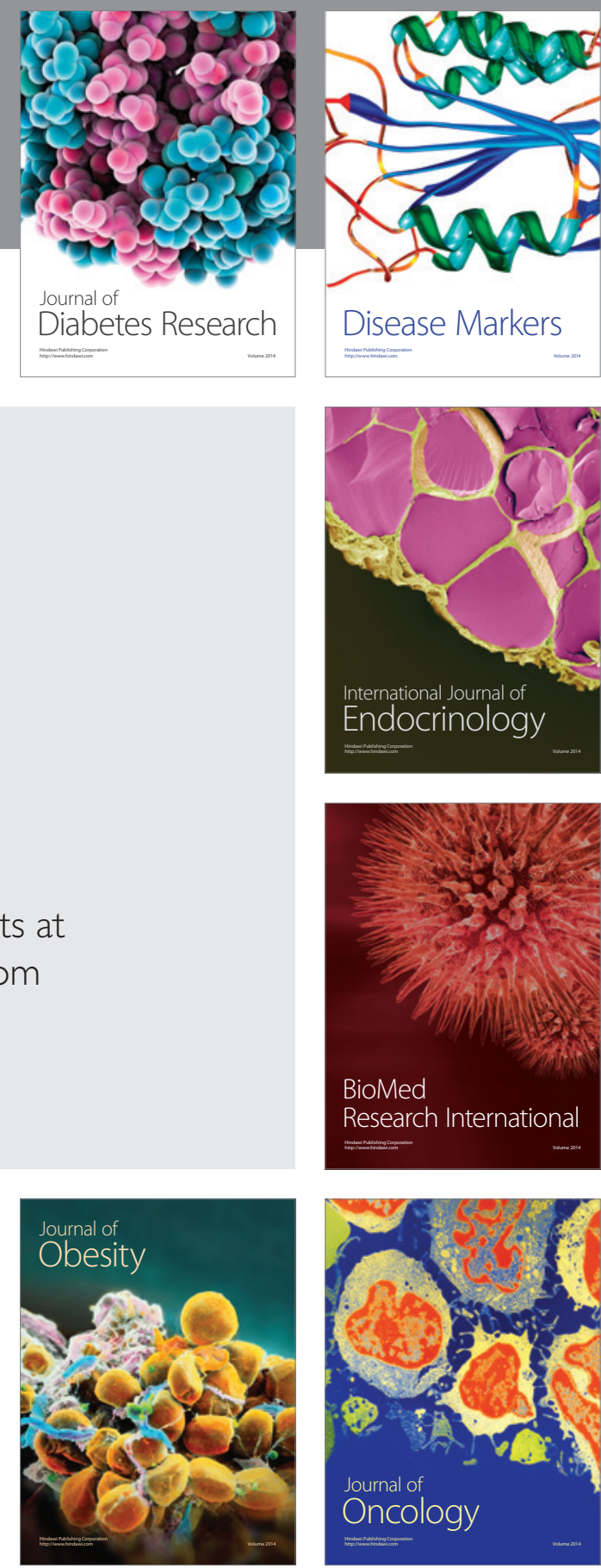

Disease Markers
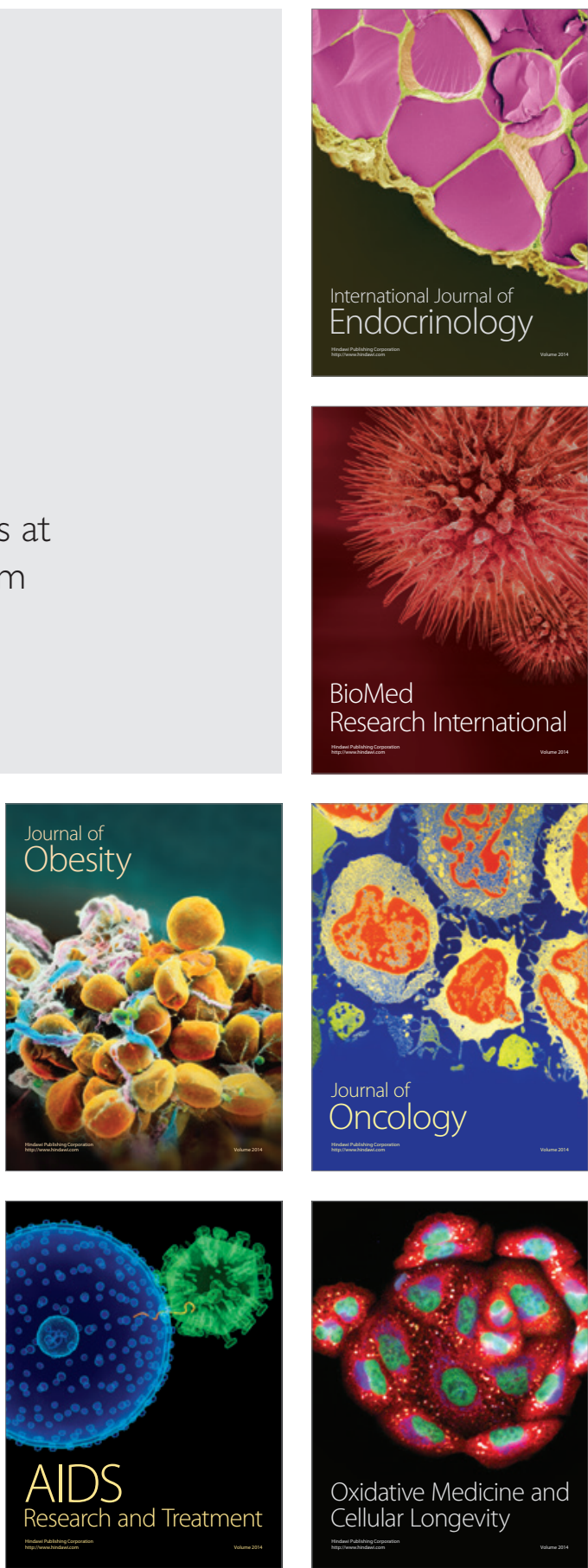